POS PROCEEDINGS

\title{
Upgrading the Prototype Schwarzschild-Couder Telescope Camera to a Wide-Field, High-Resolution Instrument
}

C. Adams $a$, G. Ambrosi $b$, M. Ambrosio $c$, C. Aramo $c$, W. Benbow $d$, B. Bertucci $b e$, E. Bissaldi $f g$, M. Bitossi $h$, A. Boiano $c$, C. Bonavolontà $c$, R. Bose $i$, A. Brill $a$, J. H. Buckley $i$, M. Caprai $b$, C. E. Covault $j$, L. Di Venere $g$, Q. Feng $k$, E. Fiandrini $b e$, A. Gent $l$, N. Giglietto $f g$, F. Giordano $f g$, R. Halliday $j$, O. Hervet $m$, G. Hughes $d$, T. B. Humensky $a$, M. Ionica $b$, W. Jin $n$, P. Kaaret $o$, D. Kieda $p$, B. $\operatorname{Kim} q$, F. Licciulli $g$, S. Loporchio $f g$, V. Masone $c$, T. Meures* $r$, B. A. W. Mode $r$, R. Mukherjee $k$, A. Okumura $s$, N. Otte $l$, F. R. Pantaleo $f g$, R. Paoletti $h t$, A. Petrashyk $a$, J. Powell $n$, K. Powell $l$, D. Ribeiro $a$, A. Rugliancich $h$, M. Santander $n$, R. Shang $q$, B. Stevenson $q$, L. Stiaccini $h t$, L. P. Taylor $r$, L. Tosti $b e$, V. Vagelli bev, M. Valentino $u c$, J. Vandenbroucke $r$, V. Vassiliev $q$, P. Wilcox $o$, D. A. Williams $m$, for the CTA SCT Project 
a Physics Department, Columbia University, New York, NY 10027, USA

b INFN Sezione di Perugia, Perugia, Italy

c INFN Napoli, Italy

d Center for Astrophysics I Harvard Smithsonian, Cambridge, MA 02138, USA

e Università degli Studi di Perugia, Perugia, Italy

f Dipartimento Interateneo di Fisica dell'Università e del Politecnico di Bari

g INFN Bari, Via E. Orabona 4, 70125 Bari, Italy

h INFN Sezione di Pisa, Pisa, Italy

i Department of Physics, Washington University, St. Louis, MO 63130, USA

j Department of Physics, Case Western Reserve University, Cleveland, Ohio 44106

$k$ Department of Physics and Astronomy, Barnard College, Columbia University, NY 10027, USA

l School of Physics \& Center for Relativistic Astrophysics, Georgia Institute of Technology, 837

State Street NW, Atlanta, GA 30332-0430, USA

$m$ Santa Cruz Institute for Particle Physics and Department of Physics, University of California,

Santa Cruz, CA 95064, USA

$n$ Department of Physics and Astronomy, University of Alabama, Tuscaloosa, AL 35487, USA

o Department of Physics and Astronomy, University of Iowa, Iowa City, IA 52242, USA

p Department of Physics and Astronomy, University of Utah, Salt Lake City, UT 84112, USA

$q$ Department of Physics and Astronomy, University of California, Los Angeles, CA, USA

$r$ Department of Physics and Wisconsin Ice Cube Particle Astrophysics Center, University of

Wisconsin, Madison, WI 53706, USA

s Institute for Space-Earth Environmental Research and Kobayashi-Maskawa Institute for the

Origin of Particles and the Universe, Nagoya University, Nagoya 464-8601, Japan

t Dipartimento di Scienze Fisiche, della Terra e dell'Ambiente, Università degli Studi di Siena,

Siena, Italy

u CNR-ISASI, Italy

v Now at ASI Italian Space Agency - Scientific Research Unit, Roma, 00133, Italy

E-mail: meures@icecube.wisc.edu

The Schwarzschild-Couder Telescope (SCT) is a candidate technology for a medium-sized telescope within the Cherenkov Telescope Array. It is expected to yield substantial improvements in field of view and image resolution compared to traditional telescopes based on Davies-Cotton optics. To match the improved optical resolution, challenging requirements of high channel count and density at low power consumption have to be met by the camera. An initial prototype camera, with 1600 pixels spanning a $2.7^{\circ}$ field of view, was installed on the prototype SCT in 2018. A project is now underway to upgrade the camera by increasing its pixel count and field of view by factors of 7 and 3, respectively (to 11,328 pixels and $8.0^{\circ}$ ). At the same time, the electronics design is being improved in order to lower the gamma-ray energy threshold and thereby provide an instrument especially well-suited for scientific studies related to extended sources and multimessenger astronomy.

36th International Cosmic Ray Conference -ICRC2019-

July 24th - August 1st, 2019

Madison, WI, U.S.A.

* Speaker. 


\section{Introduction}

The Cherenkov Telescope Array (CTA) is a planned array of Imaging Atmospheric Cherenkov Telescopes (IACTs) for detecting cosmogenic gamma rays with energies between $20 \mathrm{GeV}$ and $300 \mathrm{TeV}$ [1]. On Earth, such gamma rays can be detected via Cherenkov light emission of secondary particles, produced in the interaction of gamma rays with the atmosphere. To increase the detection probability of such particles, several telescopes will be used as an array. Stereoscopic or array configurations of IACTs also allow for more robust rejection of backgrounds. The current full CTA design consists of two array sites, one in the Northern and one in the Southern hemisphere to cover the entire sky.

Current telescope arrays, employing this technique are the High Energy Stereoscopic System (H.E.S.S.), the Major Atmospheric Gamma Imaging Cherenkov Telescopes (MAGIC) and the Very Energetic Radiation Imaging Telescope Array System (VERITAS) [2, 3, 4]. The CTA observatory is building on experience from these telescope arrays and planned to consist of three sizes of telescopes, small-, mid- and large-sized telescopes to most efficiently cover the energy range of interest.

Traditionally, Cherenkov telescopes have been constructed with a single mirror plane (DaviesCotton optics). The prototype Schwarzschild-Couder Telescope (pSCT), currently located at the Fred Lawrence Whipple Observatory (FLWO) in Arizona, is a prototype for a mid-sized telescope and has been constructed to prove a new dual-mirror optics design (Schwarzschild-Couder optics) [5]. With appropriately shaped, non-spherical mirrors, this technique allows for significantly better focusing and a greater field of view with constant camera area compared to the single mirror technique. Two other projects, utilizing Schwarzschild-Couder optics in small-sized telescopes, are the ASTRI telescope and the Gamma-ray Cherenkov Telescope (GCT) [6, 7]. The pSCT is constructed to have a field of view of $8^{\circ}$ with an angular pixel size of $0.067^{\circ}$. This improvement of the optical performance requires an approximately $0.45 \mathrm{~m}^{2}$ camera, providing a pixel size of millimeter scale.

\section{The Current pSCT Camera}

The main challenge in capturing Cherenkov images produced in the pSCT is the required pixel size of approximately $6 \mathrm{~mm} \times 6 \mathrm{~mm}$, the resulting pixel density and the timing precision of $\mathscr{O}(1 \mathrm{~ns})$. The current pSCT camera meets this challenge by using silicon photomultipliers (SiPMs) as photon sensors and the seventh generation of the TARGET chip, an application specific integrated circuit (ASIC) as the core of the front-end electronics $[8,9]$. SiPMs can be produced with millimeter pixel size for fast single photon detection and the TARGET chip is designed to sample and digitize 16 channels simultaneously at $1 \mathrm{GSa} / \mathrm{s}$; thus, the electronic footprint and power consumption are kept small, allowing the pSCT pixel density requirement to be met.

The photon sensors and readout electronics are integrated into camera modules. Modules consist of two parts, the focal plane module (FPM) and the front-end electronics (FEE). The FPM of each camera module holds 64 SiPM pixels and a Peltier cooling assembly, which allows for temperature stabilization of the SiPMs. The FEE provides control over the bias voltage supply for the SiPMs, signal shaping and digitization of the SiPM output and the temperature stabilization of the photon sensors. This part of the module holds 4 TARGET chips which perform both digitization 
and rising-edge triggering on signals from the photon channels. All functionality is controlled and monitored by a central Field Programmable Gate Array (FPGA).

The camera modules slide into the hexagonal camera body and connect to a main circuit board called backplane. The backplane handles individual power control and monitoring for up to 32 modules, as well as synchronous clock distribution and physical routing of network connections between the modules and modified network switches. Furthermore, it includes the trigger logic for combining the camera module trigger signals using a single FPGA that looks for triggers in 3 adjacent pixels within a coincidence window of a few nanoseconds. Found coincidences are latched to a $1 \mathrm{~ns}$ clock and a readout request, including the necessary timing information, is sent to all connected camera modules.

The camera body is designed to hold 177 modules which cover the entire focal plane of the pSCT optics. Currently it is equipped with 25 modules providing 1600 pixels which cover a field of view of $2.7^{\circ}$. More details on the current status of the pSCT camera can be found in [10].

\section{Planned and Ongoing Upgrades to the pSCT Camera}

The pSCT camera upgrades can be summarized as follows:

- improvements in the camera module electronics and a new generation of photon sensors,

- full population of the camera body with all 177 modules, covering the entire pSCT field of view, with consequential re-evaluation of the heat management system,

- redesign and improvement of the backplane electronics, and

- redesign of auxiliary systems.

Details on each part of the upgrade are presented below.

\subsection{The Photon Sensors and Camera Module Electronics}

The current camera is equipped with two types of photon sensors, Hamamatsu S12642-0404PA50(X) and Fondazione Bruno Kessler (FBK) NUV-HD sensors. For the camera upgrade, the third generation of the NUV-HD sensors has been chosen to equip the full camera. These sensors have multiple advantages over the currently used sensors. They exhibit an improved photon detection efficiency of greater than $50 \%$ in the relevant UV range. Furthermore, they show a reduced temperature dependence of gain and photon detection efficiency of $0.5 \%{ }^{\circ} \mathrm{C}^{-1}$.

A few more key features of the FBK NUV-HD sensors are:

- a dark rate of $150 \mathrm{kHzmm}^{-2}$, when operated with a bias voltage of $6 \mathrm{~V}$ above the specific breakdown voltage,

- a SiPM microcell area of $40 \mu \mathrm{m} \times 40 \mu \mathrm{m}$,

- optical crosstalk between microcells within a pixel of less than $20 \%$, and

- a cell recovery time of $100 \mathrm{~ns}$.

More information on the third generation of the FBK NUV-HD sensors can be found in [11].

The most significant change of the camera upgrade will be in the camera modules. In current camera modules, the photon sensors are connected to the readout electronics via $30 \mathrm{~cm}$ long microcoaxial ribbon cables. These cables are routed along the aluminum module housing, which allows a 
significant amount of electromagnetic interference to enter the cables and disturb the non-amplified SiPM signals. This problem is expected to be mitigated by using better filtering on the power regulators, moving the signal amplification circuits closer to the SiPMs, and only transmitting amplified signals via the cables.

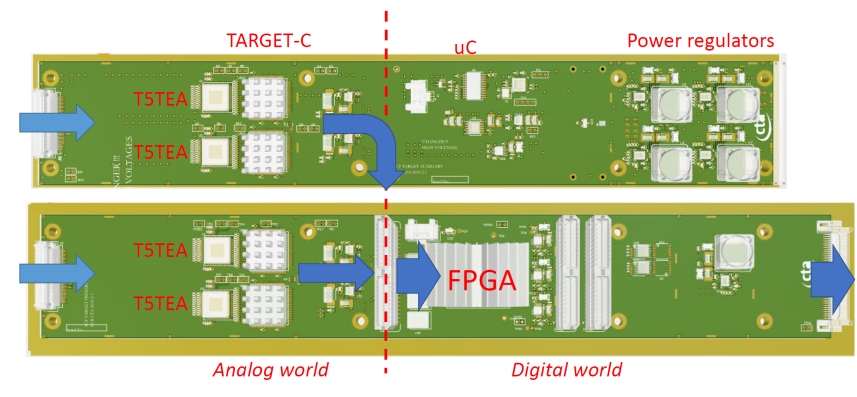

(a)

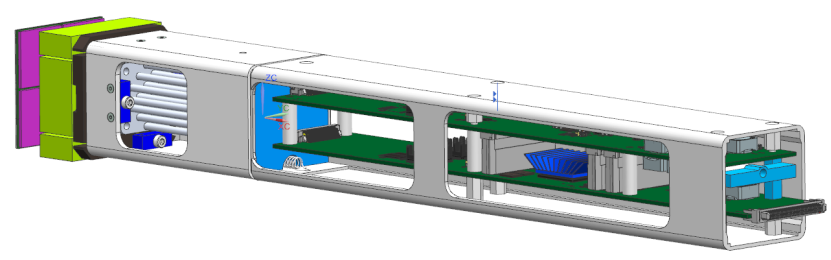

(b)

Figure 1: (a) The layout of the two electronic boards which form the upgraded camera module. (b) A 3D model of the fully assembled camera module with photon sensors and housing.

A custom ASIC, the SMART chip, is currently being developed to connect to the $16 \mathrm{SiPM}$ channels. It will provide shaping and amplification of the analog signals, as well as individual current measurements and bias voltage control for the SiPMs. The small packaging allows the chip to be deployed close to the SiPM boards and transmit the amplified signals via shorter cables to the readout electronics. This also neatens the infrastructure configuration on the two FEE boards which gives more flexibility to optimize module operation. The chip will be controlled via a Serial Peripheral Interface (SPI) bus from the central FPGA of the camera modules.

In addition, the triggering and digitization section of the module has been significantly revised. The combination of both functions in a single ASIC has proven problematic for noise performance and will be implemented separately in the upgraded camera module. The edge trigger logic will be performed by the T5TEA chip, while the digitization portion will be performed by a new generation of the TARGET chip, TARGET C [12]. This change will significantly reduce the noise on both digitized signals and the trigger, allowing for single photon triggering and resolution. The combined performance of the NUV-HD photon sensors with the first revision of the SMART ASIC and a TARGET C readout is shown in Figure 3.1. A second revision of both the SMART ASIC and the camera module with TARGET C and T5TEA are currently being fabricated and tested.

\subsection{Full camera population and associated changes}

One major step in the upgrade is to fully populate the camera body with 177 camera modules, 


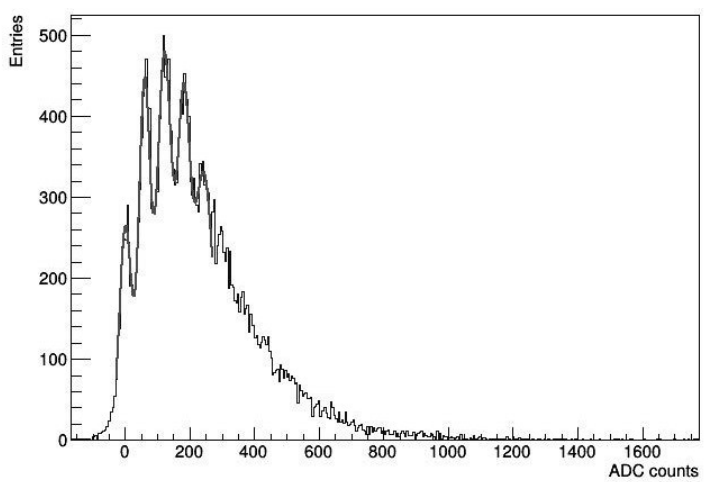

Figure 2: A photon charge spectrum, measured with the full signal chain of NUV-HD SiPM, first generation SMART ASIC and the Target C chip.

connected to 9 backplanes, and take advantage of the full field of view of the pSCT optics. This poses several challenges.

Each module consumes approximately $13 \mathrm{~W}$ of power, which leads to a combined thermal power dissipation of $2.3 \mathrm{~kW}$. This heat needs to be efficiently removed to prevent the electronics from overheating. Furthermore, it is desirable to keep the camera at a controlled constant temperature due to the temperature dependence of the SiPM gain.

The current heat management system consists of a ground chiller unit and a fan assembly on the side of the camera. The chiller unit pumps cooling fluid through a heat exchanger in front of the fan assembly at 40 gallons $/ \mathrm{min}$. The fans blow air across the heat exchanger and into the camera so that cold air is flowing through the camera body and then back to the fans in a closed loop. All camera modules are mounted in an individual aluminum housing with openings on the side which allow for air to flow through. This system has proven to be sufficient for a camera with 25 modules installed and is currently under investigation as an option for a fully equipped system of 177 modules.

Another challenge with a fully equipped camera is the timing synchronization between pixels. To properly evaluate the coincidence of photons in between pixels, they must be synchronized to a sub-nanosecond level. Such a synchronization can be achieved with standard procedures if the electronics are closely connected. However, synchronization can pose a significant challenge if clocks need to be transferred over several meters of cable. In the upgraded pSCT camera, a single backplane board will be capable of providing a clock for up to 25 modules with a precision of $\mathscr{O}(500 \mathrm{ps})$. Synchronization between backplanes is then achieved with the Distributed Intelligent Array Trigger (DIAT). The DIAT system uses a Serializer/Deserializer (SERDES) in combination with a Phased Locked Loop (PLL), to transfer trigger communications and distribute a synchronized clock of $62.5 \mathrm{MHz}$ to up to 9 backplanes. This system uses GPS provided timing and is planned to be used to interconnect telescopes and evaluate trigger coincidences between them.

\subsection{Upgrade of backplane and communication electronics}

The camera backplane in its current form has shown good performance, suitable for fulfilling the CTA requirements. A new revision needed to be produced however, since the current version was designed to accommodate 32 rather than 25 modules. The original design allowed the 
same backplane to be installed in the current pSCT camera and in CHEC (Compact High Energy Camera), a system used in small-sized two-mirror telescopes [13]. The new revision has been specifically designed for 25 camera modules so that 9 backplanes can fit in the back of the camera body. Furthermore, it includes minor changes to peripheral interfaces to optimize connections to other parts of the system.

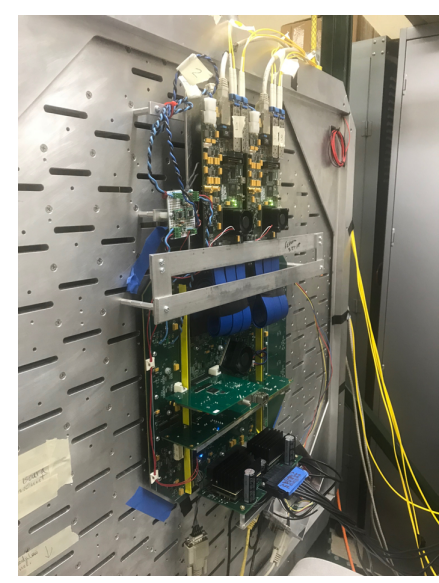

(a)

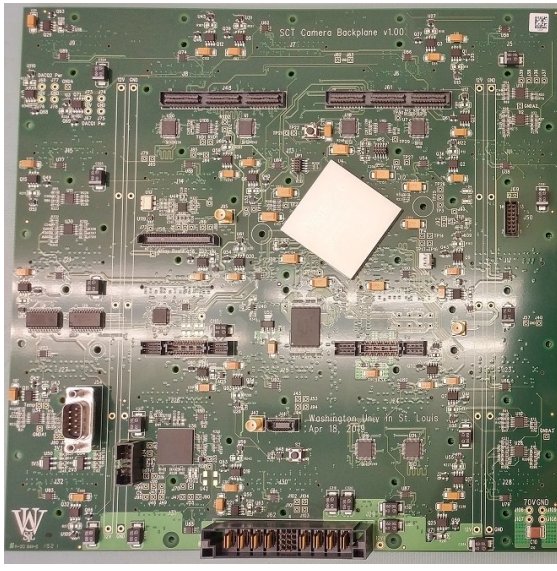

(b)

Figure 3: (a) Rear view of the current pSCT camera showing the currently installed backplane board with 32 module slots, the two DACQ boards, and peripheral connections. (b) New camera backplane, reduced to 25 slots with size allowing to distribute 9 backplanes on the rear camera body.

Communication between camera modules and a data taking server is routed via custom designed White Rabbit switches by Seven Solutions. These switches, called DACQ boards, can each interconnect 18 ports via a Gigabit link. The switches need to be upgraded with a higher transmission bandwidth (to fulfill CTA event rate requirements) and a smaller footprint (to allow for the full equipment of the camera body). The new version of the DACQ board accommodates 34 Gigabit links for the camera modules and two fiber optic links with a bandwidth of $10 \mathrm{Gbits}^{-1}$ to route the combined module data to a server. Hence only one board will be needed in the future to route all module connections. This should address both requirements, mentioned above.

\subsection{Upgrade of auxiliary systems}

Several auxiliary systems will be upgraded to enhance the performance of the camera. The camera shutter, currently a roller blinds system, will be replaced with triangular flaps in combination with a Plexiglas window to completely seal the camera from the outside atmosphere and to mitigate minor operational problems. Furthermore, the LED flashers will be upgraded to optimize their light yield and allow for synchronous triggering from the backplane.

\section{Summary and Outlook}

The pSCT camera upgrade has two main objectives: maximizing the field of view of the pSCT, and upgrading the underlying electronics. All major parts of the pSCT camera upgrade have been 
described. Camera modules will be upgraded to enhance their performance. Heat management, backplane electronics, and communication electronics will be revised to allow for the installation and operation of 177 camera modules. The current timeline for this upgrade project prioritizes the years 2019 for prototyping, 2020 for construction of subsystems, and 2021 for the integration of the subsystems and commissioning.

\section{Acknowledgements}

This work was conducted in the context of the CTA SCT Project. It is supported by National Science Foundation awards 1229792, 1828168, 1707945, and a UW 2020 award from the University of Wisconsin. Further support comes from the agencies and organizations listed in https://www.cta-observatory.org/consortium_acknowledgments/ and by the Italian Tuscany Government, POR FSE 2014 -2020, through the INFN-RT2 172800 Project. We thank these organizations for their financial contributions.

\section{References}

[1] R. A. Ong, EPJ Web Conf. 209 (2019) 01038. arXiv:1904.12196.

[2] B. Giebels, in: Proceedings, 4th International Fermi Symposium: Monterey, California, USA, October 28-November 2, 2012, 2013. arXiv:1303.2850.

[3] J. Aleksić, et al., Astroparticle Physics 72 (2016) 76-94. arXiv:1409.5594.

[4] D. Staszak, et al., 34th International Cosmic Ray Conference (ICRC2015) 34 (2015) 868. arXiv: 1510.01269.

[5] V. V. Vassiliev, et al., in: Proceedings, 30th International Cosmic Ray Conference (ICRC 2007), Vol. 3, 2007, pp. 1445-1448, [3,1445(2007)]. arXiv:0708.2741.

[6] M. C. Maccarone, et al., 35th International Cosmic Ray Conference (ICRC2017) 301 (2017) 855. arXiv: 1709.03078 .

[7] J. Dournaux, et al., Nuclear Instruments and Methods in Physics Research Section A: Accelerators, Spectrometers, Detectors and Associated Equipment 845 (2017) 355 - 358, proceedings of the Vienna Conference on Instrumentation 2016. doi:https://doi.org/10.1016/j.nima.2016.05.059.

[8] A. N. Otte, et al., Nuclear Instruments and Methods in Physics Research Section A: Accelerators, Spectrometers, Detectors and Associated Equipment 787 (2015) 85-88. doi:10.1016/j.nima.2014.11.026.

[9] A. Albert, et al., Astropart. Phys. 92 (2017) 49-61. arXiv:1607.02443.

[10] L. Taylor, et al., Camera design and performance of the prototype Schwarzschild-Couder Telescope for the Cherenkov Telescope Array, in: these proceedings, 2019.

[11] L. Tosti, et al., Development and operations of INFN optical modules for the SCT Telescope camera proposed for the CTA Observatory, in: these proceedings, 2019.

[12] S. Funk, et al., AIP Conf. Proc. 1792 (1) (2017) 080012. arXiv:1610.01536, doi:10.1063/1.4969033.

[13] R. White, Journal of Instrumentation 12 (2017) C12059. arXiv:1712.00354. 